\title{
Effects of intra-articular pulsed radiofrequency current administration on a rabbit model of rheumatoid arthritis
}

\author{
HEE KYUNG CHO ${ }^{1}$, GI-YOUNG PARK ${ }^{1}$, WOO JUNG SUNG ${ }^{2}$, SANG GYU KAWK ${ }^{3}$ and WON BIN JUNG ${ }^{4}$ \\ Departments of ${ }^{1}$ Physical Medicine and Rehabilitation, ${ }^{2}$ Pathology and ${ }^{3}$ Medical Statistics, \\ Catholic University of Daegu School of Medicine, Daegu 42472; ${ }^{4}$ Department of Physical Medicine and Rehabilitation, \\ Seongju Moo Gang Hospital, Seongju 40026, Republic of Korea
}

Received February 10, 2020; Accepted June 17, 2020

DOI: $10.3892 /$ etm.2020.9157

\begin{abstract}
Rheumatoid arthritis (RA) is a chronic autoimmune disease characterized by synovial proliferation and inflammation. Intra-articular corticosteroid injections are commonly used for the treatment of arthritis affecting one or two joints. Although corticosteroid injections are fast-acting, repeated usage can result in severe adverse events. Recently, intra-articular pulsed radiofrequency (PRF) stimulation has been proposed to treat arthritis. The aim of the present study was to compare the effectiveness of intra-articular PRF with corticosteroid injection based on histopathological and motion analysis of an ovalbumin (OVA)-induced RA rabbit model. RA was induced in the right knee joint of 18 rabbits via OVA injection. The rabbits were randomly allocated into a PRF, an intra-articular corticosteroid injection or a sham PRF stimulation group. Movement was assessed in the rabbits before treatment, then at 2,4 and 8 weeks after treatment using walking distance, fast walking time and mean walking speed. Histopathological evaluation of the distal femur and synovium was conducted 2, 4 and 8 weeks after treatment. Motion analysis demonstrated that changes in all movement variables showed significant group and time interaction as well as group effect among the three groups. The semiquantitative score based on the histopathological findings for the distal femoral condyle decreased 2 and 4 weeks after both the PRF and steroid groups, compared with the sham PRF group. Moreover, in the synovium, the semiquantitative histological score in the PRF and steroid groups tended to be lower compared with
\end{abstract}

Correspondence to: Professor Hee Kyung Cho, Department of Physical Medicine and Rehabilitation, Catholic University of Daegu School of Medicine, 33 Duryugongwon-ro 17-gil, Nam, Daegu 42472, Republic of Korea

E-mail:hkcho@cu.ac.kr

Abbreviations: RA, rheumatoid arthritis; PRF, pulsed radiofrequency; OVA, ovalbumin; NSAIDs, nonsteroidal anti-inflammatory drugs

Key words: RA, ovalbumin-induced arthritis, intra-articular, PRF, corticosteroid, histopathological, motion analysis the sham PRF group, although this result was not statistically significant. Thus, intra-articular PRF stimulation may delay cartilage destruction and improve functional motion in RA.

\section{Introduction}

Rheumatoid arthritis (RA) is a painful and disabling polyarticular autoimmune disease (1). In recent decades it has been estimated that $\leq 1 \%$ of the global population is affected by RA (1). This disease is characterized by proliferation and inflammation of the synovium, which is the membrane lining the inner surface of the joint capsule. RA progression leads to the formation of the pannus, a layer of fibrovascular or granulation tissue, which causes progressive degradation of the adjacent articular cartilage, ligaments and bone (2-6). The normal synovial lining is 2-4 layers thick. However, in RA, the synovial lining forms a layer 10-15 cells deep. This tissue is then infiltrated with macrophages, B cells, mast cells, fibroblast-like synoviocytes and $\mathrm{CD} 4^{+} \mathrm{T}$ lymphocytes, which contribute to synovial inflammation and joint destruction $(4,7)$. First-line therapy for RA consists of reducing synovial inflammation and controlling pain, which primarily comprises the use of medication, such as nonsteroidal anti-inflammatory drugs (NSAIDs), disease-modifying antirheumatic drugs and oral corticosteroids (8). Although various medications are used orally, some joints often remain refractory to this type of treatment. In general, intra-articular corticosteroid injections are considered effective an treatment for inflamed focal synovitis and are widely and conventionally used for the management of RA joint pain. However, because of possible negative effects, this type of treatment is controversial. Although the immediate ameliorating results of local administration are clear, the long-term effects of corticosteroid injections remain unknown. In addition, corticosteroids can have adverse effects, such as allergic reactions, flushing, hyperglycemia, immunosuppression, menstrual changes and adrenal suppression $(9,10)$.

Sluijter et al (11) introduced an isothermal radiofrequency treatment called pulsed radiofrequency (PRF) stimulation, which can be applied to specific nerves causing neuropathic pain. It has been suggested that the electric field rather than the temperature generated by PRF is responsible for the clinical effects of this treatment (11). Notably, this treatment does not 
substantially destroy nerves and tissues. PRF therapy uses a brief stimulation followed by a long resting phase and exposes the target tissue to a high electric field around an electrode tip and shaft without producing sufficient heat to cause structural damage (11). Due to its minimally destructive effects on neural tissues, PRF stimulation has been further developed and rapidly adopted in clinical practice. More recently, the effectiveness of PRF has encouraged certain clinicians to use this method in the intra-articular space to manage arthritic pain (12-14). Previous studies have reported that applying intra-articular PRF into the knee joints ameliorates the severity of the disease in addition to reducing swelling, exudates in joint fluid and damage to cartilage $(12,13)$. Schianchi et al $(14)$ suggested that intra-articular PRF may induce significant long-term pain relief in patients with joint pain. Patients with intractable shoulder, knee, trapezio-metacarpal and first metatarso-phalangeal joint pain were treated with intra-articular PRF and the reported success rate appeared to be high in small joints. However, few clinical studies have investigated the effects of intra-articular PRF on arthritic pain (12-14), and there are no reports on PRF treatment using an animal RA model.

In the present study, antigen-induced RA was established in rabbits, as described by Wollheim et al (15). This method induces arthritis that manifests in only one joint and exhibits many histological similarities to RA in humans. The aim of the present study was to investigate the effects of intra-articular PRF stimulation on pain-related behavior using motion analysis and histopathological evaluation in the synovium and cartilage of this RA rabbit model.

\section{Materials and methods}

Animals. New Zealand white rabbits (18; age, 12-weeks; weight, 2.0-2.5 kg) were provided (Daehan Biolink Co., Ltd.) for the present study. The rabbits were housed in cages at room temperature with 40-60\% humidity under a 12-h light-dark cycle with free access to food and water. After adaptive feeding for 1 week, RA was induced in the rabbits using ovalbumin (OVA) injection, as described by Wollheim et al (15). Briefly, $20.0 \mathrm{mg} / \mathrm{ml}$ OVA solution was prepared using phosphate-buffered saline at $\mathrm{pH} 7.4$, mixed with equal volumes of Complete Freund's Adjuvant, and sufficiently emulsified at $4^{\circ} \mathrm{C}$. An injection of $1.0 \mathrm{ml}$ OVA solution containing $10.0 \mathrm{mg}$ OVA was subcutaneously injected into five areas between the scapular region of the rabbit, and two additional weekly injections were likewise administered to strengthen the immune response. RA was induced on week 5 by injecting $0.5 \mathrm{ml}$ of the OVA solution containing $5.0 \mathrm{mg}$ OVA into the right articular cavity.

Pulsed radiofrequency administration procedures. The rabbits were randomly divided into three groups ( $n=6$ in each group) as follows: i) Intra-articular PRF administration group; ii) sham stimulation group; and iii) intra-articular corticosteroid injection group (steroid). Four weeks after the introduction of RA, the rabbits received either intra-articular PRF administration, sham stimulation, or a corticosteroid injection. An intramuscular injection of $15 \mathrm{mg} / \mathrm{kg}$ Zoletil (tiletamine hydrochloride and zolazepam hydrochloride; Virbac) and $5 \mathrm{mg} / \mathrm{kg}$ Rompun (xylazine; Bayer AG) compound was administered to anesthetize the animals (16). PRF was administered by placing an electrode (Cosman RFG-1A generator and RF Thermocouple Electrode; $500 \mathrm{~mm}$; CoMedical) into the articular cavity of the right knee using ultrasound guidance with a 5-13 MHZ multifrequency linear transducer (Antares; Siemens Healthcare), as displayed in Fig. 1. A 5-Hz PRF current was administered for $120 \mathrm{sec}$ at $45 \mathrm{~V}$. The left knee was used as a control. The present PRF protocol is based on clinical studies on the application of intra-articular PRF $(14,17)$. For rabbits in the sham PRF group, the electrode was placed in precisely the same manner, with the instrument turned off so that PRF stimulation was not applied to the articular cavity. In the steroid group, $1 \mathrm{mg}$ triamcinolone in a $0.1 \mathrm{ml}$ volume was injected into the articular cavity of the right knee using ultrasound guidance.

Motion analyses. Rabbits with ovalbumin-induced arthritis received motion analysis before treatment, then 2, 4 and 8 weeks after treatment. All animals were allowed to acclimatize in an open space for $30 \mathrm{~min}$ before their movements were analyzed. The rabbits were then left to move freely in a $3 \times 3 \mathrm{~m}$ space for $5 \mathrm{~min}$ and their horizontal movements were recorded using a camera equipped with a SMART video-tracking observation system (version 3.0.03; Panlab; Harvard Bioscience, Inc.). The movements were measured using walking distance, fast walking time and mean walking speed (18).

Histopathological analysis. To compare the effects of PRF stimulation and the steroid injection on the RA joint cavity, two rabbits from each group were sacrificed 2, 4 and 8 weeks after receiving PRF or steroid treatment. Both the distal femurs and surrounding synovium were removed and fixed in $4 \%(\mathrm{w} / \mathrm{v})$ paraformaldehyde for $24 \mathrm{~h}$ at room temperature. The femurs were also decalcified with $10 \%$ nitric acid solution for $24 \mathrm{~h}$ at room temperature. The decalcified femur and synovium that had not undergone the decalcification process were embedded in paraffin for sectioning at a thickness of $4 \mu \mathrm{m}$. Hematoxylin and eosin (H\&E) staining was performed using an automated slide stainer (Sakura ${ }^{\circledR}$ Tissue-Tek $^{\circledR}$ DRS $^{\text {TM }}$ 2000) for 8 min of hematoxylin and $5 \mathrm{~min}$ of eosin staining at room temperature. For Toluidine blue staining, sections were equilibrated in 95\% alcohol, and then stained with solution of toluidine blue for $10 \mathrm{~min}$ at room temperature. The histological sections were observed under a light microscope (magnification, $\mathrm{x} 100$ and $\mathrm{x} 200$ ) and a semiquantitative histopathological score for the distal femurs was assigned according to the method of Cake et al (19), using a five-point scale based on structure $(0-10)$, cellularity (0-4), chondrocyte cloning (0-4), territorial toluidine blue staining (0-4) and interterritorial toluidine blue staining (0-4). A mean aggregate score was determined as the average of these values, ranging between 0 (normal) and 26 (19). Additionally, the morphological parameters of synovitis were assessed using a light microscope (magnification, x200) according to the method of Krenn et al (20) as follows: i) Hyperplasia/enlargement of synovial lining layer; ii) degree of inflammatory infiltration; and iii) activation of resident cells/synovial stroma. All parameters were graded as 0 (absent), 1 (slight), 2 (moderate) or 3 (strong positive), and the scores ranged from 0 (no synovitis) to 9 . One blinded pathologist who did not have any information about the intervention evaluated and scored the slides. 

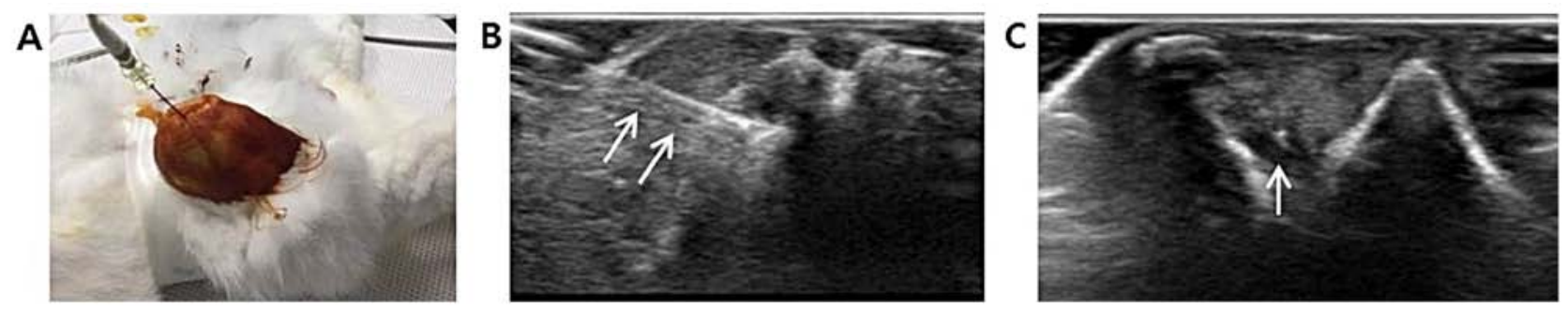

Figure 1. PRF administration under ultrasound guidance. (A) A PRF needle was placed into the articular cavity of the right knee. (B) The PRF needle was observed as a hyperechoic line on a longitudinal image and (C) a small hyperechoic dot on a transverse image. PRF, pulsed radiofrequency.
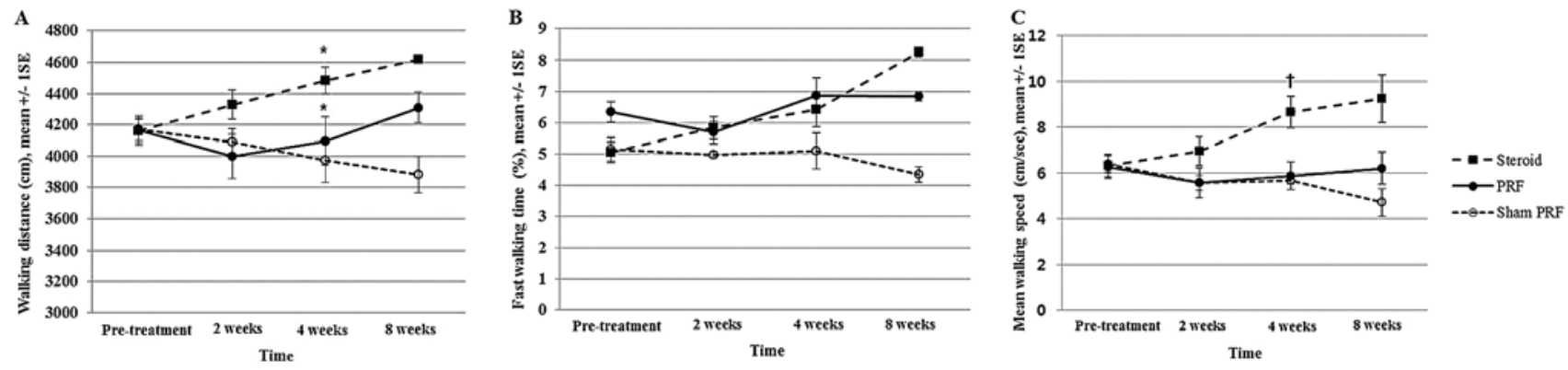

Figure 2. Motion analysis. Motion analysis of ovalbumin-induced arthritis in rabbits pre-treatment and 2, 4, and 8 weeks after intra-articular PRF administration, corticosteroid injection (steroid), and sham PRF. (A) Walking distance (cm). (B) Fast walking time (\%). (C) Mean walking speed (cm/s). ${ }^{*} \mathrm{P}<0.05$, vs. sham PRF group; ${ }^{\top} \mathrm{P}<0.05$, vs. PRF and sham PRF groups. PRF, pulsed radiofrequency; 1SE, one standard error.

Statistical analysis. Motion analysis and histopathological data from the intra-articular PRF, steroid, and sham PRF groups were analyzed using the Kruskal-Wallis test, followed by Dunn's post hoc test. Changes in motion analysis according to time, group, and the combined effect of time/group were analyzed using a univariate Generalized Linear Model (GLM), and multiple comparisons were conducted using simple contrast. A medical statistician conducted data analysis using SPSS version 19.0 (IBM Corp.). All tests were two-sided and $\mathrm{P}<0.05$ was considered to indicate a statistically significant difference.

\section{Results}

Motion analysis. Motion analyses were conducted to assess the effect of PRF or corticosteroid treatments on movement over time. Analysis of fast walking time showed significant time effect $(\mathrm{P}=0.021)$, group effect $(\mathrm{P}<0.001)$, and group and time interaction $(\mathrm{P}=0.003)$. Analysis of walking distance and mean walking speed showed no significant time effect $(\mathrm{P}>0.05)$. However, there was a significant group effect $(\mathrm{P}<0.001)$ and group and time interaction $(\mathrm{P}<0.05)$ in both walking distance and mean walking speed. Indeed, walking distance significantly differed in both the PRF and steroid groups, compared with the sham PRF group 4 weeks after treatment $(P=0.048$, PRF vs. sham PRF group; $\mathrm{P}=0.018$, steroid vs. sham $\mathrm{PRF}$ group). In addition, the steroid group exhibited significantly faster movements, compared with the PRF and sham PRF groups at 4 weeks $(\mathrm{P}=0.031$, steroid vs. $\mathrm{PRF}$ group, $\mathrm{P}=0.023$, steroid vs. sham PRF group). However, fast walking time at 2, 4 and 8 weeks after treatment did not differ across the three groups (Fig. 2).
Histopathological analysis. The distal femurs and surrounding synovium were observed 2, 4 and 8 weeks after treatment. $\mathrm{H} \& \mathrm{E}$ staining of the distal femurs indicated severe fibrillation and/or erosion of the cartilage in the sham PRF group. The sham group also displayed a severe decrease in territorial and interterritorial toluidine blue staining of the distal femurs, indicating loss of articular cartilage integrity. In both the PRF and steroid groups, surface irregularities with clefts of the cartilage were observed (Fig. 3). Histopathological score for the distal femurs demonstrated significantly lower score at 2 and 4 weeks after treatment in the PRF and steroid groups compared with in the sham PRF group $(\mathrm{P}<0.05$; Table I).

$\mathrm{H} \& \mathrm{E}$ staining of the synovium displayed strong inflammatory infiltration of lymphatic follicles and/or confluent subsynovial lymphatic infiltration with hyperplasia of the synovial cell layer in the sham PRF group. However, inflammatory infiltration of the synovium was reduced in the PRF and steroid groups, compared with the sham group (Fig. 4). Histopathological score for the synovium suggested that the PRF and steroid groups tended to have lower score for synovitis than the sham PRF group, although the difference among the three groups was not statistically significant (Table II).

\section{Discussion}

The present study suggested that both intra-articular PRF administration and corticosteroid injection may improve functional walking distance as well as histopathological results for the distal femurs in an OVA-induced RA rabbit model.

RA is a common systemic autoimmune disease that causes chronic inflammation in the joints and other organs, particularly in the synovial membranes and articular structures (1). 

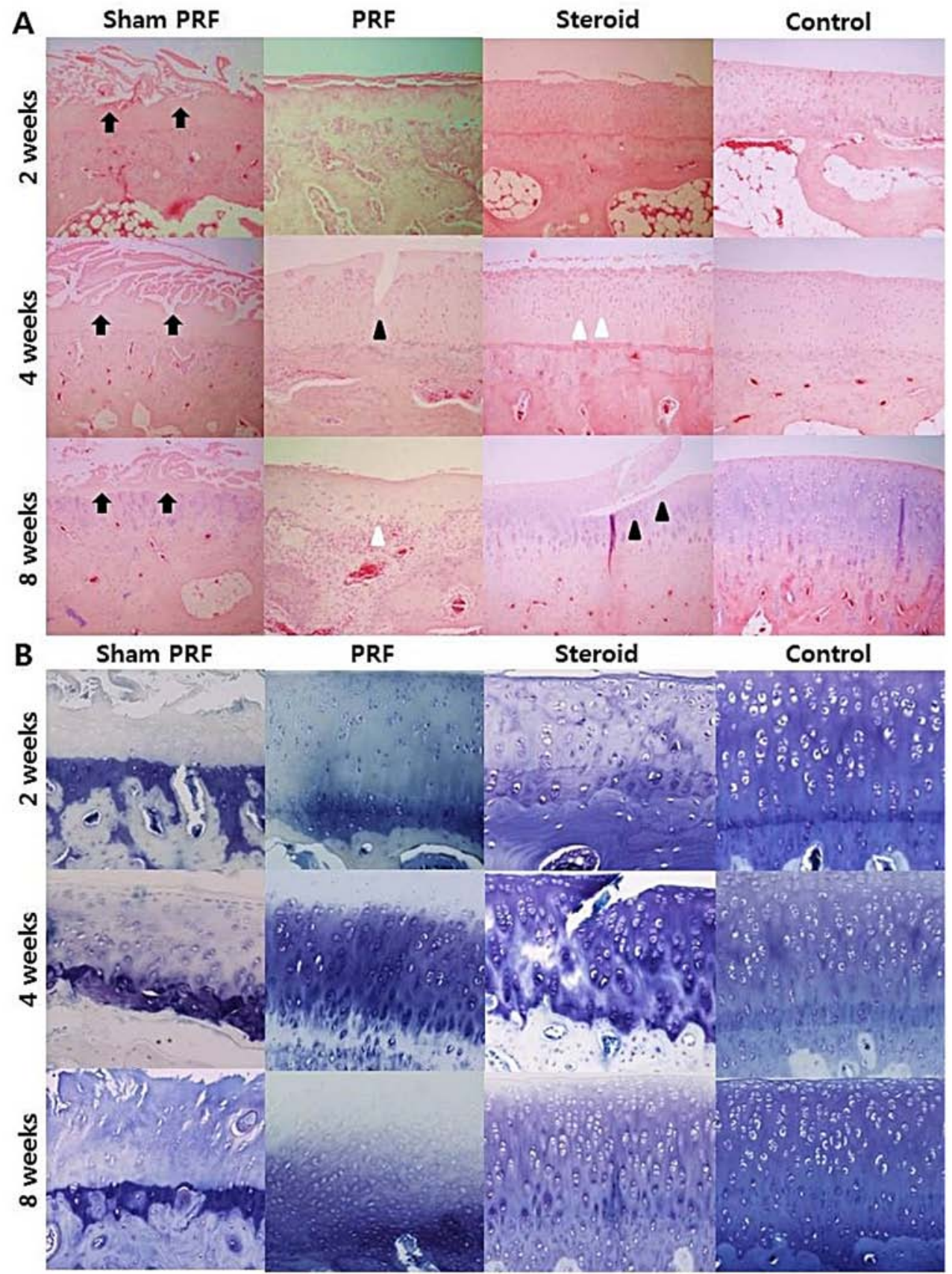

Figure 3. Histopathological staining of distal femurs 2, 4 and 8 weeks after intra-articular PRF administration, corticosteroid injection (steroid) and sham PRF. Opposite, non-arthritic knees were used as negative controls. (A) Histopathological staining with hematoxylin and eosin. Fibrillation and/or erosion in the calcified zone are indicated in the sham PRF group (black arrows) and some clefts/fissures with surface irregularities are visible in the PRF and steroid groups (black arrowheads). Decreased cellularity and chondrocyte doublets are detectable in the PRF and steroid groups (white arrowheads). Magnification, x100. (B) Histopathological staining with toluidine blue. Severe decreased territorial toluidine blue staining in the sham group and slight-to-moderate decreased territorial toluidine blue staining in the PRF and steroid groups. Magnification, x200. PRF, pulsed radiofrequency.

RA pathogenesis typically involves excess synovial fluid, synovial cell hyperplasia and formation of a pannus, which can eventually damage the articular cartilage and cause joint deformities $(5,6)$. Previous studies have suggested that RA is partly the result of inflammation within the joint that results in high levels of proinflammatory cytokines $(21,22)$. Thus, RA treatment strategies usually include the use of anti-inflammatory drugs directed at cytokines to prevent the progression of structural changes within the joint. Although various oral medications are used, focal joint edema often occurs, which causes a painful condition in patients with RA. In general, corticosteroids suppress various inflammatory cytokines and chemokines, and intra-articular corticosteroid injections are commonly administered to patients with RA-related pain.

Previous studies have indicated that intra-articular triamcinolone hexacetonide is effective in reducing synovitis (15) and joint destruction (23) in antigen-induced arthritis. The present study also used an antigen-induced arthritis animal model of mono-articular disease that affects only the injected joints. Histopathological findings in this model exhibit similarities 
Table I. Histopathological scores for the distal femurs 2, 4 and 8 weeks after intra-articular PRF administration, corticosteroid injection (steroid) or sham PRF.

Time

\begin{tabular}{lccc}
\cline { 2 - 4 } Group & 2 weeks & 4 weeks & 8 weeks \\
\hline Control & $4.000 \pm 0.584$ & $4.625 \pm 0.519$ & $4.458 \pm 0.513$ \\
Sham PRF & $15.875 \pm 1.546^{\mathrm{a}}$ & $16.25 \pm 0.968^{\mathrm{a}}$ & $16.750 \pm 0.323^{\mathrm{a}}$ \\
PRF & $12.000 \pm 2.051^{\mathrm{a}, \mathrm{b}}$ & $13.250 \pm 1.963^{\mathrm{a}, \mathrm{b}}$ & $14.250 \pm 1.451^{\mathrm{a}}$ \\
Steroid & $10.375 \pm 0.747^{\mathrm{a}, \mathrm{b}}$ & $12.250 \pm 1.785^{\mathrm{a}, \mathrm{b}}$ & $8.125 \pm 1.008^{\mathrm{a}-\mathrm{c}}$ \\
\hline
\end{tabular}

Values are the mean $\pm \mathrm{SD}$. PRF, pulsed radiofrequency. ${ }^{\mathrm{a}} \mathrm{P}<0.05$ vs. control group, ${ }^{\mathrm{b}} \mathrm{P}<0.05$ vs. sham $\mathrm{PRF}$ group, ${ }^{\mathrm{c}} \mathrm{P}<0.05$ vs. $\mathrm{PRF}$ group.

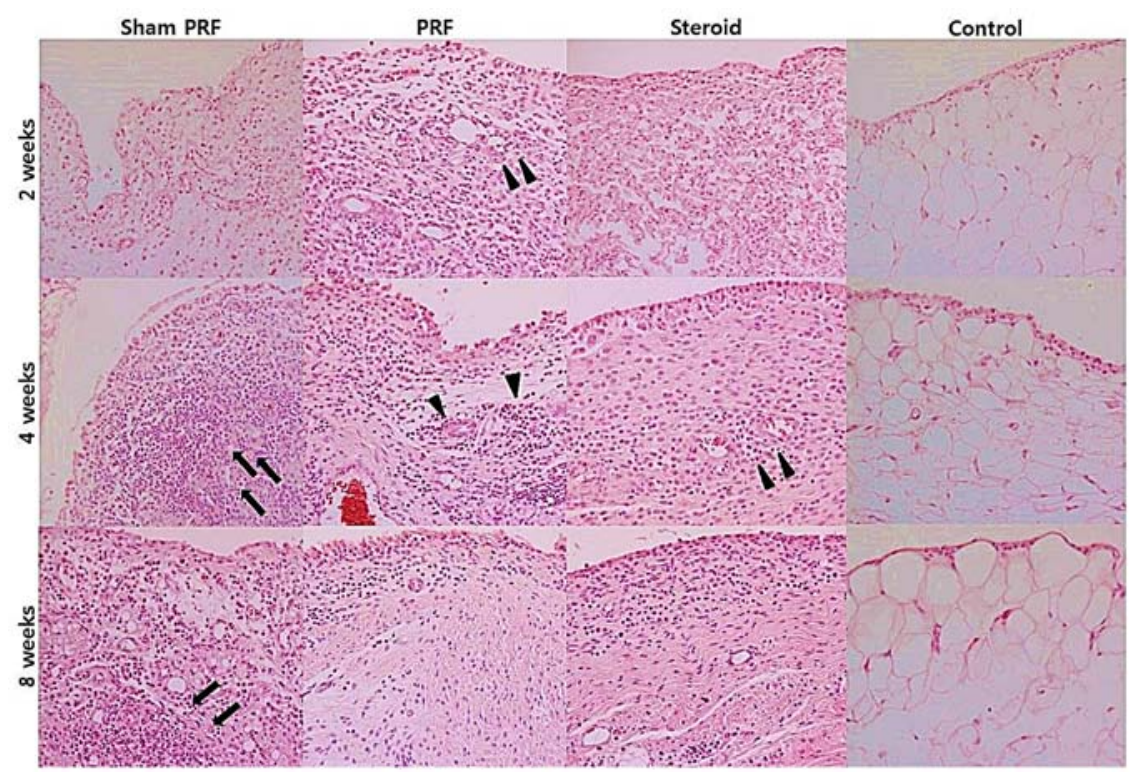

Figure 4. Histopathological staining with hematoxylin and eosin of the synovium 2, 4 and 8 weeks after intra-articular PRF administration, corticosteroid injection (steroid) and sham. Opposite, non-arthritic knees were used as negative controls. Major enlargement of the synovial lining cell layer with lymphatic follicles and/or confluent subsynovial lymphatic infiltration was observed in the sham group (black arrows). Moderate-to-major enlargement of the synovial lining cell layer was observed in the PRF and steroid groups. Slight-to-moderate inflammatory infiltration (small perivascular and/or superficial lymphatic aggregates) was observed in the PRF and steroid groups (black arrowheads). Magnification, x200. PRF, pulsed radiofrequency.

to RA in humans, including synovial hyperplasia, inflammatory infiltration of lymphatic follicles, pannus formation and cartilage erosion (24). In the present study, histopathological features were improved in rabbits treated with corticosteroids. Nevertheless, the effect of corticosteroid treatment for RA remains controversial. Although local corticosteroid treatment has a marked and immediate ameliorating effect, repeated corticosteroid injections can result in severe complications, such as septic arthritis $(25,26)$. Considering the side effects observed with corticosteroid treatment, the aim of the present study was to determine the effectiveness of intra-articular PRF administration in RA joints. The findings of the present study were not notable enough to consider PRF clearly superior to steroid treatment, since no significant intergroup difference was determined based on histopathological scoring of the distal femur 2 and 4 weeks after treat ment. However, the PRF group displayed improved motion and histopathological findings for the distal femur, compared with the sham PRF group.
PRF has initially been used as an alternative to continuous RF for the treatment of peripheral nerves causing neuropathic pain (27-29). Although the mechanisms underlying the effects of PRF stimulation have not been fully elucidated, it has been suggested that the electrical field produced by PRF can alter pain signals $(30,31)$. Van Zundert et al (30) revealed that c-fos immunoreactive neurons were increased in the superficial laminae of the spinal dorsal horn after PRF administration to the dorsal root ganglion. Hagiwara et al (31) demonstrated that PRF may actually enhance the descending noradrenergic and serotonergic inhibitor pathway, which are involved in the neuropathic pain. Due to its advantages as a minimally neurodestructive approach, PRF is also used for other types of pain, in addition to neuropathic pain. For instance, it has been reported that PRF treatment can effectively relieve discomfort from painful joints if the needle is placed within the joint $(12-14,17,32)$. The present study also indicated an improving trend in pain-related behavior following intra-articular PRF administration, compared with the sham PRF group. 
Table II. Histopathological scores for the synovium 2, 4 and 8 weeks after intra-articular PRF administration, corticosteroid injection (steroid) or sham PRF.

Histopathological score at each timepoint

\begin{tabular}{lccc}
\cline { 2 - 4 } Group & 2 weeks & 4 weeks & 8 weeks \\
\hline Control & $1.917 \pm 0.664$ & $1.333 \pm 0.401$ & $1.917 \pm 0.455$ \\
Sham PRF & $5.750 \pm 1.250^{\mathrm{a}}$ & $6.500 \pm 0.000^{\mathrm{a}}$ & $6.250 \pm 0.250$ \\
PRF & $4.500 \pm 1.000^{\mathrm{a}}$ & $5.250 \pm 0.750^{\mathrm{a}}$ & $5.250 \pm 1.750$ \\
Steroid & $4.500 \pm 0.500^{\mathrm{a}}$ & $4.750 \pm 0.250^{\mathrm{a}}$ & $3.000 \pm 0.500^{\mathrm{a}}$ \\
\hline
\end{tabular}

Values are the mean $\pm \mathrm{SD}, \mathrm{PRF}$, pulsed radiofrequency. ${ }^{\mathrm{a}} \mathrm{P}<0.05$ vs. control group.

In particular, there was a significant increase in walking distance at 4 weeks after PRF administration, compared with the sham PRF group. It was hypothesized that this difference may be caused by the effect of PRF on the nervous system. Indeed, joints are innervated by the peripheral nerve branches entering the joint capsule. Many simple nerve endings are located at the attachments of joint capsules and are believed to be terminals of unmyelinated and thinly myelinated nociceptive axons (33). Moreover, direct influence of an electric field can inhibit the excitability of pain-generating nerves or free nerve endings $(14,17,27,28,30)$. Erdine et al (34) applied PRF to the afferent axons of the sciatic nerves of rats and observed the internal ultrastructural components of axons using electron microscopy. It was found that the axons showed microscopic damage after PRF exposure. The damage was more pronounced from the $\mathrm{C}$-fibers to the A $\mathrm{A}$ fibers to the $\mathrm{A} \beta$ fibers following PRF administration. This result suggested that PRF might have a more pronounced effect on smaller pain-carrying fibers (C-fiber and A $\delta$ fibers), and a lesser effect on larger $A \beta$ fibers mediating non-pain-related sensations (34). Thus, it may be suggested that intra-articular PRF could disrupt the synovial lining nociceptive $\mathrm{C}$-fibers, for instance.

Unlike previous clinical studies, the present study also investigated the differences in histopathological findings after intra-articular PRF administration, compared with a sham PRF group, as well as in rabbits receiving corticosteroid treatment. The histopathological findings demonstrated a significant decrease in damage to the medial and lateral femoral condyles 2 and 4 weeks after intra-articular PRF administration or corticosteroid injection, compared with the sham PRF group. The current findings supported another possible mechanism of PRF on the immune system $(35,36)$. The histopathological findings of the present study suggested decreased damage to the distal femurs. Although these results were not significant for the synovium, the microscopic view during the study suggested decreased infiltration of inflammatory cells into the synovium after treatment in the PRF and steroid groups compared with the sham PRF group. Although RA pathogenesis is still poorly understood, activated $\mathrm{T}$ cells, $B$ cells and macrophages invading the joint synovium involved in the pathogenesis of RA (37). These inflammatory cells release various proinflammatory cytokines and mediators, which cause severe tissue damage and secondary inflammatory injuries in RA (38). It has been hypothesized that an electric field from a PRF may affect immune cells, as there are studies that show proinflammatory cytokines, such as TNF $\alpha$, are affected by electric fields $(35,36)$. Fini et al suggested that pulsed electric field delivery combines a chondrocyte proliferation, matrix synthesis effect and anti-inflammatory action in osteoarthritis (39). Therefore, the resulting decreased cytokine levels in the joint microenvironment may, in turn, favor intrinsic and extrinsic cartilage repair (17).

The limitations of the present study primarily include the small sample size. Moreover, only the functional and histopathological manifestations of RA were assessed. To evaluate pain-related behavior, motion analysis was also conducted, which, to the best of the authors' knowledge, has not been attempted in previous studies. Although this analysis allowed adequate functional assessment of rabbit movements, non-arthritic rabbits were not included as controls. Moreover, to determine whether PRF affects the immune system and reduces RA-related inflammation and cartilage destruction, additional studies involving immunohistochemical examination are required. Lastly, the lack of standardized methods for the administration of intra-articular PRF in rabbits implies that results may have been influenced by the administration protocol. The present study was designed to apply a single PRF administration 4 weeks after RA induction. Although the infiltration of inflammatory cells into the synovium tended to be decreased in the PRF group compared to the sham PRF group, synovial hyperplasia was only marginally reduced in the PRF group in comparison to the sham PRF group. This may suggest that the present treatment protocol could be modified in order to further improve the observed effects on synovial hyperplasia.

In conclusion, intra-articular PRF administration can ameliorate pain-related behavior, delay cartilage destruction and attenuate synovial inflammation. It is hypothesized that intra-articular PRF affects the nociceptive nerve fibers of the synovial lining, inhibits the transfer of pain signals from the nerves into the joint capsule and reduces the inflammatory response associated with RA. To the best of the authors' knowledge, the present study is the first to evaluate the functional and histopathological effects of intra-articular PRF administration in a rabbit model of RA. Additional studies involving a larger number of cases and various PRF administration protocols are required to address the limitations of the present study. 


\section{Acknowledgements}

Not applicable.

\section{Funding}

The present study was supported by The National Research Foundation of Korea grant funded by the Korean government (Ministry of Science and Information and Communications Technologies; grant no. 2016R1C1B1014185).

\section{Availability of data and materials}

The datasets used and/or analyzed during the current study are available from the corresponding author on reasonable request.

\section{Authors' contributions}

HKC conceived the study. HKC, WJS, SGK and WBJ developed the methodology. SGK analyzed the data. HKC, WJS and WBJ interpreted the data and drafted the manuscript. HKC and GYP validated the analysis and reviewed the manuscript. All authors read and approved the final manuscript.

\section{Ethics approval and consent to participate}

The present study protocol was approved by the Animal Research Ethics Committee of Daegu Catholic University (approval no. DCIAFCR-170131-2-Y).

\section{Patient consent for publication}

Not applicable.

\section{Competing interests}

The authors declare that they have no competing interests.

\section{References}

1. Alamanos Y and Drosos AA: Epidemiology of adult rheumatoid arthritis. Autoimmun Rev 4: 130-136, 2005.

2. Firestein GS: Evolving concepts of rheumatoid arthritis. Nature 423: 356-361, 2003.

3. Garnero P, Landewe R, Boers M, Verhoeven A, Van Der Linden S, Christgau S, Van Der Heijde D, Boonen A and Geusens P: Association of baseline levels of markers of bone and cartilage degradation with long-term progression of joint damage in patients with early rheumatoid arthritis: The COBRA study. Arthritis Rheum 46: 2847-2856, 2002.

4. Kim KR, Chung TY, Shin H, Son SH, Park KK, Choi JH and Chung WY: Red ginseng saponin extract attenuates murine collagen-induced arthritis by reducing pro-inflammatory responses and matrix metalloproteinase-3 expression. Biol Pharm Bull 33: 604-610, 2010.

5. Rhee DK, Marcelino J, Baker M, Gong Y, Smits P, Lefebvre V, Jay GD, Stewart M, Wang H, Warman ML and Carpten JD: The secreted glycoprotein lubricin protects cartilage surfaces and inhibits synovial cell overgrowth. J Clin Invest 115: 622-631, 2005.

6. Cassim B, Shaw OM, Mazur M, Misso NL, Naran A, Langlands DR, Thompson PJ and Bhoola KD: Kallikreins, kininogens and kinin receptors on circulating and synovial fluid neutrophils: Role in kinin generation in rheumatoid arthritis Rheumatology (Oxford) 48: 490-496, 2009.
7. Tristano AG: Tyrosine kinases as targets in rheumatoid arthritis. Int Immunopharmacol 9: 1-9, 2009.

8. Kumar LD, Karthik R, Gayathri N and Sivasudha T: Advancement in contemporary diagnostic and therapeutic approaches for rheumatoid arthritis. Biomed Pharmacother 79: 52-61, 2016.

9. Manchikanti L: Role of neuraxial steroids in interventional pain management. Pain Physician 5: 182-199, 2002.

10. Manchikanti L, Boswell MV, Singh V, Benyamin RM, Fellows B, Abdi S, Buenaventura RM, Conn A, Datta S, Derby R, et al: Comprehensive evidence-based guidelines for interventional techniques in the management of chronic spinal pain. Pain Physician 12: 699-802, 2009.

11. Sluijter ME, Cosman ER, Rittman WB and van Kleef M: The effects of pulsed radiofrequency fields applied to the dorsal root ganglion: A preliminary report. Pain Clin 11: 109-117, 1998.

12. Masala S, Fiori R, Raguso M, Morini M, Calabria E and Simonetti G: Pulse-dose radiofrequency for knee osteoartrithis. Cardiovasc Intervent Radiol 37: 482-487, 2014.

13. Karaman H, Tufek A, Kavak GO, Yildirim ZB, Uysal E, Celik F and Kaya S: Intra-articularly applied pulsed radiofrequency can reduce chronic knee pain in patients with osteoarthritis. J Chin Med Assoc 74: 336-340, 2011.

14. Schianchi PM, Sluijter ME and Balogh SE: The treatment of joint pain with intra-articular pulsed radiofrequency. Anesth Pain Med 3: 250-255, 2013.

15. Wollheim FA, Telhag H, Henricsson A and Geborek P: Prevention of joint destruction in antigen-induced arthritis. Clin Immunol Immunopathol 70: 19-21, 1994.

16. Karasu A, Altug N, Aslan L, Bakir B and Yuksek N: Evaluation of the anesthetic effects of xylazine-ketamine, xylazine-tiletamine-zolazepam and tiletamine-zolazepam using clinical and laboratory parameters in rabbits. Med Weter 74: 646-652, 2018.

17. Sluijter ME, Teixeira A, Serra V, Balogh S and Schianchi P: Intra-articular application of pulsed radiofrequency for arthrogenic pain-report of six cases. Pain Pract 8: 57-61, 2008.

18. Park GY, Kwon DR and Lee SC: Regeneration of full-thickness rotator cuff tendon tear after ultrasound-guided injection with umbilical cord blood-derived mesenchymal stem cells in a rabbit model. Stem Cells Transl Med 4: 1344-1351, 2015.

19. Cake MA, Read RA, Guillou B and Ghosh P: Modification of articular cartilage and subchondral bone pathology in an ovine meniscectomy model of osteoarthritis by avocado and soya unsaponifiables (ASU). Osteoarthritis Cartilage 8: 404-411, 2000.

20. Krenn V, Morawietz L, Haupl T, Neidel J, Petersen I and Konig A: Grading of chronic synovitis-a histopathological grading system for molecular and diagnostic pathology. Pathol Res Pract 198: 317-325, 2002.

21. Kastelein RA, Hunter CA and Cua DJ: Discovery and biology of IL-23 and IL-27: Related but functionally distinct regulators of inflammation. Annu Rev Immunol 25: 221-242, 2007.

22. Wilson NJ, Boniface K, Chan JR, McKenzie BS, Blumenschein WM, Mattson JD, Basham B, Smith K, Chen T, Morel F, et al: Development, cytokine profile and function of human interleukin 17-producing helper T cells. Nat Immunol 8: 950-957, 2007.

23. Hunneyball IM: Some further effects of prednisolone and triamcinolone hexacetonide on experimental arthritis in rabbits. Agents Actions 11: 490-498, 1981.

24. Choudhary N, Bhatt LK and Prabhavalkar KS: Experimental animal models for rheumatoid arthritis. Immunopharmacol Immunotoxicol 40: 193-200, 2018.

25. Ostensson A and Geborek P: Septic arthritis as a non-surgical complication in rheumatoid arthritis: Relation to disease severity and therapy. Br J Rheumatol 30: 35-38, 1991.

26. Xing D, Yang Y, Ma X, Ma J, Ma B and Chen Y: Dose intraarticular steroid injection increase the rate of infection in subsequent arthroplasty: Grading the evidence through a meta-analysis. J Orthop Surg Res 9: 107, 2014.

27. Cho HK, Cho YW, Kim EH, Sluijter ME, Hwang SJ and Ahn SH: Changes in pain behavior and glial activation in the spinal dorsal horn after pulsed radiof requency current administration to the dorsal root ganglion in a rat model of lumbar disc herniation: Laboratory investigation. J Neurosurg Spine 19: 256-263, 2013.

28. Park HW, Ahn SH, Son JY, Kim SJ, Hwang SJ, Cho YW and Lee DG: Pulsed radiofrequency application reduced mechanical hypersensitivity and microglial expression in neuropathic pain model. Pain Med 13: 1227-1234, 2012.

29. Choi GS, Ahn SH, Cho YW and Lee DK: Short-term effects of pulsed radiofrequency on chronic refractory cervical radicular pain. Ann Rehabil Med 35: 826-832, 2011. 
30. Van Zundert J, de Louw AJ, Joosten EA, Kessels AG, Honig W, Dederen PJ, Veening JG, Vles JS and van Kleef M: Pulsed and continuous radiofrequency current adjacent to the cervical dorsal root ganglion of the rat induces late cellular activity in the dorsal horn. Anesthesiology 102: 125-131, 2005.

31. Hagiwara S, Iwasaka H, Takeshima $\mathrm{N}$ and Noguchi T: Mechanisms of analgesic action of pulsed radiofrequency on adjuvant-induced pain in the rat: Roles of descending adrenergic and serotonergic systems. Eur J Pain 13: 249-252, 2009.

32. Ozyuvaci E, Akyol O, Acikgoz A and Leblebici H: Intraarticular pulsed mode radiofrequency lesioning of glenohumeral joint in chronic shoulder pain: 3 cases. Korean J Pain 24: 239-241, 2011.

33. Chen C, Lu Y, Kallakuri S, Patwardhan A and Cavanaugh JM: Distribution of A-delta and C-fiber receptors in the cervical facet joint capsule and their response to stretch. J Bone Joint Surg Am 88: 1807-1816, 2006.

34. Erdine S, Bilir A, Cosman ER and Cosman ER Jr: Ultrastructural changes in axons following exposure to pulsed radiofrequency fields. Pain Pract 9: 407-417, 2009.

35. Sluijter ME and Imani F: Evolution and mode of action of pulsed radiofrequency. Anesth Pain Med 2: 139-141, 2013.
36. Maretto F, Vennik M, Albers KI and van Duijn B: TNFa secretion of monocytes exposed to pulsed radiofrequency treatment: A possible working mechanism of PRF chronic pain management. Pain Pract 14: 399-404, 2014.

37. Hu Y, Cheng W, Cai W, Yue Y, Li J and Zhang P: Advances in research on animal models of rheumatoid arthritis. Clin Rheumatol 32: 161-165, 2013.

38. Chun J, Tosun A and Kim YS: Anti-inflammatory effect of corymbocoumarin from Seseli gummiferum subsp. corymbosum through suppression of NF- $\mathrm{B}$ B signaling pathway and induction of HO-1 expression in LPS-stimulated RAW 264.7 cells. Int Immunopharmacol 31: 207-215, 2016.

39. Fini M, Giavaresi G, Carpi A, Nicolini A, Setti S and Giardino R: Effects of pulsed electromagnetic fieds on articular hyaline cartilage: Review of experimental and clinical studies. Biomed Pharmacother 59: 388-394, 2005.

This work is licensed under a Creative Commons

Attribution-NonCommercial-NoDerivatives 4.0 International (CC BY-NC-ND 4.0) License. 